\section{A Patient with Massive Pneumocephalus without Sign of Tension Pneumocephalus}

\section{Abstract}

Background: Pneumocephalus is an injury that characterized by air entrance into the intracranial space. It may occur after head trauma, infection, tumor or it may be iatrogenic. According to the amount of air increases, this can cause neurological findings with a mass effect and this condition is called tension pneumocephalus.

Case report: In this study present a case of a 63 year old man presented to the emergency department by EMS because of head trauma. He was found to have loss of consciousness. Brain CT-scan revealed the typical Mount Fuji sign suggestive of massive pneumocephalus. The patient was transferred to the ICU and was managed conservatively without any decompressive surgery. On evaluation of 5 days later, he was alert and asymptomatic and control brain CT-scan has minimal pneumocephalus. Then, the patient was discharged from hospital.

Conclusion: Although massive pneumocephalus is a complication that if it not treated immediately, it may be convert to tension pneumocephalus and give rise to serious morbidity and mortality concerns, but this case showed massive pneumocephalus without sign of tension pneumocephalus that conservative treatment was done successfully.

Keywords: Head trauma; Massive pneumocephalus; Conservative management
Seyed Farshad Heidari

Mazandaran University of Medical Sciences, Sari, Mazandaran, Iran

Corresponding author:

Seyed Farshad Heidari

झ s.f.heidari@gmail.com

Mazandaran University of Medical Sciences, Sari, Mazandaran, Iran.

Tel: 988338224670

Fax: 9188317379

Citation: Heidari SF. A Patient with Massive Pneumocephalus without Sign of Tension Pneumocephalus. J Intensive \& Crit Care 2016, 2:3.

\section{Introduction}

Pneumocephalus is a pathology that is defined by the presence of air into the intracranial region. It is usually secondary to trauma, infection and tumor or it may be iatrogenic [1]. In general, this is due to a bone defect allowing communication between the intracranial compartment and one of the cranial sinuses. Tension pneumocephalus is a condition in which pneumocephalus progresses and giving a mass effect by creating intracranial hypertension and leading to neurological disorders [2] and neurosurgical intervention is documented to be a clinical setting associated with an increased risk of this complication [3].

\section{Case Report}

A 63 year old man presented to the emergency department by EMS because of head trauma. The incidence of pedestrian with car was mechanism of trauma. Patient had nausea, vomiting and rhinorrheagia. On physical examination, he was found to have headache, agitation, confusion, PTA with GCS of 14/15. Vital sign was stable. There was bilateral periorbital ecchymosis and one laceration with size of $2 \mathrm{~cm}$ in left eyebrow. Remnant examinations was unremarkable. Axial images of the brain on a computed tomographic scan (CT-scan) showed an extensive pneumocephalus in frontal on both sides without midline shift. Also, there was evidence of fracture in left maxillary sinus and skull base fracture (Figure 1). The patient was transferred to the ICU and treated conservatively with head elevation 30 deg and oxygen therapy. On evaluation of 5 days later, he was alert and asymptomatic. Control brain CT-scan has minimal pneumocephalus so that numerous amount of pneumocephale was absorbed. Then, the patient was discharged from hospital with related recommendations.

\section{Discussion}

Head trauma is the most common cause of pneumocephalus ( $80 \%$ of cases approximately) [4] that occurs in 0.5 to $1 \%$ of cases of head trauma [5]. Besides of head traumas, it is seen after neurosurgical operations, cerebellopontine angle tumors, shunt operations, post-barotrauma, post-lumbar puncture and etc. [5-7]. 


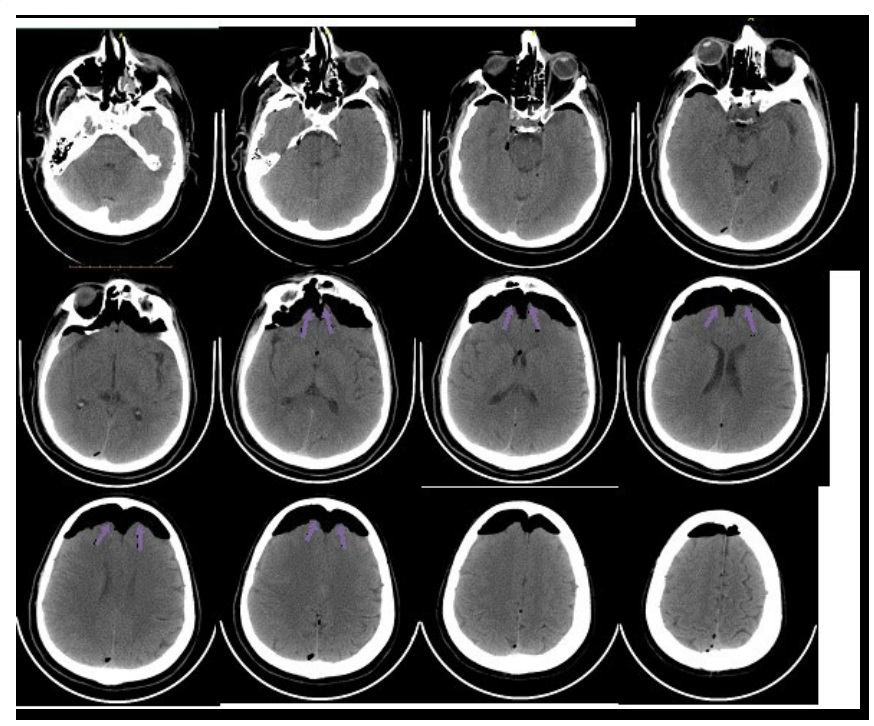

Figure 1 Brain CT-scan of a patient with bulky pneumocephalus. Axial CT-scan of brain showing pneumocephalus causing compression of bilateral frontal lobes with separation of their tips and resultant twin peaks (arrows) signifying Mount Fuji Sign.

The mechanism of formation of pneumocephalus has been explained by the ball-valve mechanism [8] and the inverted-sodabottle effect [9]. After injury to the skull base, conditions such as coughing, sneezing, vomiting induce positive pressure that cause air to enter into the skull from outside via a defect and lead to tension pneumocephalus. In tension pneumocephalus, clinical presentation is in the form of headache, seizures, agitation, delirium, reflex abnormalities, cognitive abnormalities and brain stem herniation in serious cases (changes in rhythm, hypertension, cranial nerve paralyses, bradycardia and cardiac arrest) [10]. Therefore, it is of extreme clinical importance to differentiate a tension pneumocephalus from a non-tension pneumocephalus as the latter does not usually require decompressive surgery.

CT-scan is the gold standard diagnostic tool for pneumocephalus that identifies the presence of air via the density differences between air, fluid, tissue and bone. CT-scan is used to identify the amount and location of air and fluid, presence of a defect and the effect of trapped air on the brain [11]. Mount Fuji sign is characterized by large air collections over bilateral frontal lobes which cause compression of frontal lobes and separation of the tips of the frontal lobes typically forming twin peaks. The characteristic separation of the tips of the frontal lobes indicates that the tension of the air exceeds the surface tension of the CSF between the frontal lobes. Although Mount Fuji sign are proposed as most specific for tension pneumocephalus, and an important sign to differentiate it from non-tension pneumocephalus that, necessitate emergent decompression, but it is not Indicative of tension pneumocephalus in all cases, unless it is associated with detoriation of clinical status $[12,13]$. In this case, there was Mount Fuji sign associated with stable clinical status that was demonstrator of massive pneumocephalus without sign of tension pneumocephalus.

Pneumocephalus usually resolves by itself and progresses asymptomatically. However, if the amount of air entering into the skull increases and creates a mass effect, this is called tension pneumocephalus. This is a pathology that is rarely seen but it may induce cerebellum herniation by creating a mass effect [14]. Neurological findings depend on the amount of air, the air leakage rate and its location. They occur when the amount of air exceeds $25 \mathrm{cc}$, as a result of various compensation mechanisms of the brain [15].

In patients with pneumocephalus, the first line of treatment is conservative. In this treatment, the bed head is elevated and it is requested that the patient remains still and abstains from maneuvers that will cause an increase in intra-sinus pressure such as sneezing, coughing, valsalva, etc. Wide spectrum antibiotic therapy is started because of the risk of meningitis. After this treatment, pneumocephalus undergoes resolution in some patients and recovery may be possible. Based on previous recommendations, if findings of tension pneumocephalus appear, this is an indication for immediate neurosurgical intervention that a burr hole should be opened and the defect in the skull base should be closed as soon as possible [16].

Our case report is implied to the fact that although the posttraumatic brain CT-scan showed the typical Mount Fuji sign with compression and characteristic separation of tips of the frontal lobes, but the patient was clinically stable and showed no neurological deterioration (bulky pneumocephalus without sign of tension pneumocephalus) and patient was managed conservatively instead of surgical intervention. There was almost complete resolution of the pneumocephalus in the follow up CTscan after 5 days. Then the patient was discharged from hospital with necessitous recommendations.

\section{Conclusion}

Although massive pneumocephalus is a complication if it is not treated immediately, it may give rise to serious morbidity and mortality concerns, but this case showed that aggressive treatment will not need in all cases. 


\section{References}

1 Schirmer CM, Heilman CB, Bhardwaj A (2010) Pneumocephalus: case illustrationsand review. Neurocrit Care 13: 152-158.

2 Dowd GC, Molony TB, Voorhies RM (1998) Spontaneous otogenic pneumocephalus. Case report and review of the literature. J Neurosurg 89: 1036-1039.

3 Vaquero J, Cabeaudo JM (1981) Late postoperative tension pneumatocoele. J Neurosurg 55: 145-146.

4 Toung TJK, McPherson RW, Ahn H (1986) Pneumocephalus: Effects of patient position on the incidence and location of aerocele after posterior fossa and upper cervical cord surgery. Anesth Analg 65: 65-70.

5 Lee SH, Koh JS, Bang JS (2009) Extensive tension pneumocephalus caused by spinal tapping in patient with basal skull fracture and pneumothorax. J Korean Neurosurg Soc 45: 318-321.

6 Vikram A, Deb AK (2011) Tension pneumocephalus secondary to a dural tear. Appl Radiol 40: 37-38.

7 Schnipper D, Spiegel JH (2004) Management of intracranial complications of sinus surgery. Otolaryngol Clin North Am 37: 453-472.

8 Horowitz M (1964) Intracranial pneumocele. An unusual complication following mastoid surgery. J Laryngol Otol 78: 128-134.

9 Kuncz A, Roos A, Lujber L (2004) Traumatic prepontine tension pneumocephalus: Case report. Ideggyogy Sz 57: 313-315.

10 Sadler DJ, Doyle GJ, Hall K (1996) Cranio-cervical bone pneumatisation. Neuroradiology 38: 330-332.

11 Webber-Jones JE (2005) Tension pneumocephalus. J Neurosci Nurs 37: $272-276$.

12 Yamashita S, Tsuchimochi W, Yonekawa T (2009) The Mount Fuji Sign on MRI. Intern Med 48: 1567-1568.

13 Ishiwata Y, Fujitsu K, Sekino T, Fujino H, Kubokura T, et al. (1988) Subdural tension pneumocephalus following surgery for chronic subdural hematoma. J Neurosurg 68: 58-61.

14 North JB (1999) On the importance of intracranial air. Br J Surg 34: 345-349.

15 DelGaudio JM, Ingley AP (2010) Treatment of pneumocephalus after endoscopic sinus and microscopic skull base surgery. Am J Otolaryngol 31: 226-230.

16 Prüss H, Klingebiel R, Endres M (2011) Tension pneumocephalus with diplegia and deterioration of consciousness. Case Rep Neurol 14: 48-49. 